\title{
Precise gene deletion and replacement using the CRISPR/Cas9 system in human cells
}

\section{PROTOCOL For: \\ Precise gene deletion and replacement using the CRISPR/Cas9 system in human cells}

Qiupeng Zheng ${ }^{1}$, Xiaohong Cai², Meng How Tan, ${ }^{3,4}$, Steven Schaffert², Christopher P. Arnold², Xue Gong'², Chang-Zheng Chen ${ }^{2,5}$, and Shenglin Huang ${ }^{1,2}$

${ }^{1}$ Fudan University Shanghai Cancer Center, Institute of Biomedical Sciences, and Department of Oncology, Shanghai Medical School, Fudan University, Shanghai, China, ${ }^{2}$ Baxter Laboratory for Stem Cell Biology, and Department of Microbiology and Immunology, Stanford University School of Medicine, Stanford, CA, ${ }^{3}$ School of Chemical and Biomedical Engineering, Nanyang Technological University, Singapore, ${ }^{4}$ Agency for Science Technology and Research, Genome Institute of Singapore, Singapore, ${ }^{5}$ Achelois Pharmaceuticals, Inc., San Francisco, CA

\section{BioTechniques Protocol Guide, January 2017; doi 10.2144/000114508}

See full protocol online: https://benchtalk.biotechniques.com/channels/276-molecular-biology-genome-engineering-protocol-community/ posts/6416-protocol-for-precise-gene-deletion-and-replacement-using-the-crispr-cas9-system-in-human-cells

Protocol Summary: Here, we describe targeted gene deletion and replacement in human cells via the CRISPR/Cas9 system using two guide RNAs. The system effectively generated targeted deletions of varied length, regardless of the transcriptional status of the target gene. It is notable that targeted gene deletions generated via CRISPR/Cas9 and two guide RNAs resulted in the formation of correct junctions at high efficiency. Moreover, in the presence of a homology repair donor, the CRISPR/Cas9 system could guide precise gene replacement. Our results illustrate that the CRISPR/Cas9 system can be used to precisely and effectively generate targeted deletions or gene replacement in human cells, which will facilitate characterization of functional domains in protein-coding genes as well as noncoding regulatory sequences in animal genomes.

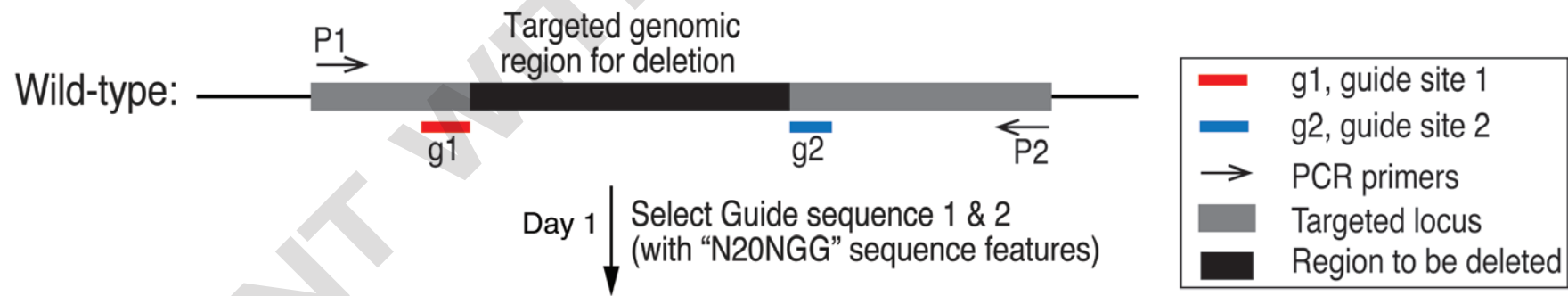

\section{Deleted:}

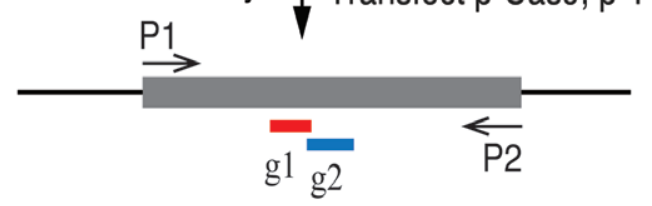

Day $7 \mid \begin{gathered}\text { PCR and Sequencing } \\ \text { Validation }\end{gathered}$

Protocol Schematic: The steps of targeted gene deletion with CRISPR/Cas9. 